\title{
Démarches de Développement La Participation des Habitants
}

\author{
Michel Bonetti * \\ Patrice Séchet ${ }^{* *}$
}

\begin{abstract}
$\mathrm{R}$ participation des habitants que nous avons expérimenté dans le cadre de différentes opérations de réhabilitation ou de réaménagement urbain de quartiers d'habitat social.

La maîtrise d'ouvrage de ces opérations était assurée par des organismes de logement social ou des collectivités locales.

Après avoir précisé les différentes formes de participation que l'on peut mettre en œuvre, nous présentons les principes qui doivent sous-tendre selon nous la mise en place des processus de participation.

Nous développons ensuite la conception de ces processus qui nous paraît la plus pertinente et la plus efficace, et à l'issue de cet exposé méthodologique nous illustrons nos propos par un exemple concret de processus de participation que nous avons contribué à mettre en œuvre à la demande de l'OPAC de Paris, dans le cadre d'une opération de réaménagement de l'espace urbain d'un quartier de $\mathbf{7 0 0}$ logements.
\end{abstract}

Palavras-Chave: participation des habitants; opérations de réhabilitation; logement social.

\section{La Clarification des Formes et des Objectifs de la Participation}

La notion de participation est particulièrement confuse. Il importe de clarifier les objectifs que l'on poursuit et de ne pas leurrer les habitants sur l'influence qu'ils peuvent avoir sur les décisions. On peut distinguer quatre formes d'implication des habitants dans la conduite des projets:

\section{L'information}

Les habitants sont simplement informés des projets, ils ne participent pas à leur élaboration et au processus de décisions.

\section{La consultation}

Le fait de consulter les habitants revient à prendre leur avis. Cela signifie que le maître d'ouvrage s'efforcera de les prendre en compte, mais il ne s'engage pas pour autant à le faire. La consultation n'implique pas la mise en place d'une négociation. Ceci doit être clairement explicité, afin de ne pas créer de malentendus et de déceptions.
Les habitants peuvent être consultés avant l'élaboration d'un projet pour connaître leurs attentes, ou on peut solliciter leur avis sur un avant-projet, afin d'en tenir compte pour mettre au point le projet définitif.

Les consultations a posteriori pour connaître l'avis des habitants sur des projets entièrement définis à l'avance ne visent généralement qu'à entériner les décisions déjà prises. Elles sont perçues comme une manipulation, et font perdre toute crédibilité aux maîtres d'ouvrage.

\section{La concertation}

La mise en place d'une concertation suppose que les décideurs s'engagent à négocier avec les habitants. Il importe alors de préciser:

- L'objet de la concertation, ce qui est négociable et ce qui ne l'est pas;

- Les modalités de la négociation (avec qui, comment);

- Les contraintes et les limites de la négociation, et notamment ce qui n'est pas négociable.

\footnotetext{
* Director do Laboratoire de Sociologie Générative Urbaine, CSTB.Contacto: pagliarini@cstb.fr

** Investigador do Laboratoire de Sociologie Générative Urbaine, CSTB.Contacto: pagliarini@estb.fr
} 


\section{La coopération}

L'engagement d'une coopération avec des groupes d'habitants suppose que ceux-ci sont associés étroitement, de bout en bout, à l'élaboration d'un projet. Les habitants impliqués participent alors au travail technique de définition du projet et aux décisions.

Il importe de préciser là aussi quel est l'objet de la coopération, qui peut concerner certaines parties seulement d'un projet global (aménagement de halls, d'aires de jeux, élaboration de règles de vie collectives, un mode de traitement des réclamations, etc...).

Règles à respecter:

- Il ne faut jamais faire des promesses que l'on ne peut pas tenir car on perd toute crédibilité. Il ne faut jamais promettre d'associer les habitants à la décision, si on n'est pas sûr de pouvoir tenir ses engagements.

- Il vaut mieux mettre en place une bonne information ou organiser une concertation efficace plutôt que promettre une fausse coopération dans laquelle on engage un simulacre.

\section{La Conception des Processus de Participation}

Nous proposons un modèle d'organisation de la participation des habitants qui articule une instance de décision, une équipe opérationnelle et une instance d'expression de la demande.

Dans la conduite de la plupart des projets les acteurs distinguent généralement:

- Une instance de décision assurant la maîtrise d'ouvrage des projets;

- Une équipe opérationnelle qui réalise les diagnostics, élabore les programmes et les projets et assurent leur mise en œuvre après validation par l'instance de décision.

Nous proposons la mise en place d'une «instance d'expression de la demande» conformément au schéma suivant.

Le rôle de l'équipe opérationnelle consiste à:

- Organiser l'expression de la demande et la négociation avec les habitants;
- Intégrer autant que faire se peut les demandes des habitants, et à faire des propositions qu'ils soumettent aux décideurs après les avoir interprétées et hiérarchisées (cf. ci-après).

Figure 1 - Mis en place d'une «instance d'expression de la demande»

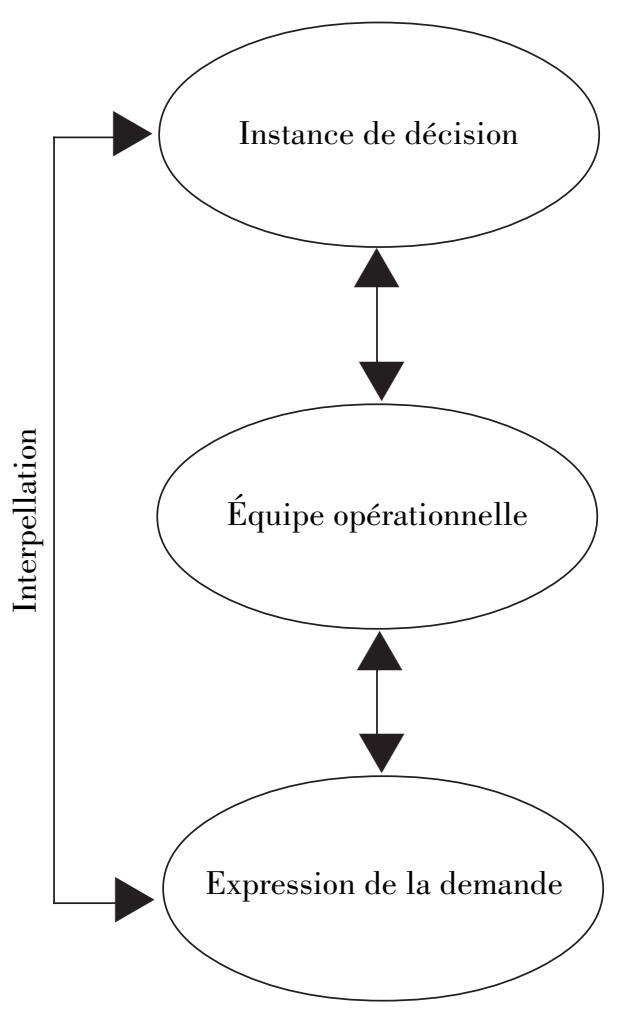

Dans ce modèle les habitants ne sont pas intégrés à l'instance de décision, mais associés au processus de décision. Il appartient aux décideurs de se positionner par rapport aux demandes exprimées par les habitants et aux solutions préconisées par l'équipe opérationnelle pour y répondre.

Les habitants ne sont donc pas intégrés à l'instance de décision, mais en contre-partie cela nécessite que:

- Les décideurs expriment la volonté de favoriser l'expression et la prise en compte de leur demande;

- Le dispositif de conduite de projet traduise concrètement cette volonté;

- L'équipe opérationnelle organise cette expression de la demande et la négociation avec les habitants en ayant le souci d'intégrer les attentes des habitants dans l'élaboration des projets;

- Que les décideurs explicitent leurs choix et 
acceptent en retour d'être interpellés sur leurs décisions et le cas échéant de les modifier.

Il s'agit donc de mettre en tension l'instance de décision et l'expression de la demande dont l'articulation est assurée par l'équipe opérationnelle qui organise et met en scène ces relations.

Établir une Distinction claire entre les Décideurs et les Habitants pour Eviter la Confusion des Rôles et des Responsabilités

Nous ne proposons pas d'intégrer les habitants dans l'instance de décision (alors que d'autres le préconisent) pour plusieurs raisons. Tout d'abord l'expérience montre que les dispositifs dans lesquels les habitants sont censés participer directement aux instances de décision aboutissent à ce que les décisions soient prises ailleurs, dans le cadre de réunions limitées aux décideurs. Il y a donc un risque très fort de manipulation, qui ne peut qu'entraîner la suspicion des habitants qui se sentent floués. C'est souvent le cas des comités de quartier ou des comités de pilotage ou les habitants sont censés avoir le même statut que les principaux décideurs.

En outre la participation des habitants à des instances de décision ne peut se faire que par le truchement de représentants qui, bien que légitimes, risquent de ne pas toujours prendre en comptes les attentes de l'ensemble des habitants (notamment des jeunes, des immigrés et des groupes les plus marginalisés).

Nous considérons que les décideurs sont soit élus pour assumer une responsabilité politique, soit désignés pour assumer la responsabilité économique d'une organisation (cas des directeurs d'organismes HLM), responsabilités que n'ont pas les habitants.

Il importe donc que les décideurs assument pleinement leurs responsabilités économiques ou politiques pour lesquelles ils ont été élus ou désignés. Ils doivent prendre en compte différents facteurs que les habitants directement concernés par les projets n'intègrent pas : l'intérêt des habitants de l'environnement, le développement urbain de la collectivité locale, la pérennité économique des organismes de logements, les coûts de maintenance, etc.
Mais en contre-partie les décideurs doivent s'efforcer de connaître et de prendre en compte les attentes des habitants et de débattre des choix auxquels ils aboutissent.

Les Problèmes Soulevés par les Habitants et leurs Attentes sont Pleinement Légitimes, mais il Appartient aux Professionnels d'Élaborer des Solutions Pertinentes

Nous n'acceptons pas la position des professionnels qui prétendent connaître les problèmes et qui refusent d'écouter les difficultés que ressentent les habitants et de prendre en compte leurs attentes. L'expérience montre que cette position aboutit à la réalisation d'espaces proprement invivables.

Nous nous démarquons également des acteurs qui pensent a contrario que les professionnels doivent se contenter de reprendre et de traduire les solutions préconisées par les habitants.

Les habitants sont parfaitement légitimes et pertinents quand ils expriment leurs problèmes et leurs attentes, et ils peuvent faire des suggestions judicieuses qu'il convient d'examiner attentivement, mais il ne sont généralement pas en mesure de construire un projet résolvant la majeure partie des problèmes auxquels ils sont confrontés et intégrant les multiples contraintes qu'un projet doit gérer.

Les solutions qu'ils envisagent sont liées à leur connaissance sur le sujet, qui peuvent être limitées et à leurs représentations. Elles peuvent parfois accroître les problèmes dont ils souffrent et s'avérer en contradiction avec leurs propres attentes.

A partir d'une écoute et d'une compréhension fine des problèmes et des attentes exprimées par les habitants, il appartient aux professionnels de déconstruire ces problèmes, d'interpréter ces attentes et d'élaborer des esquisses de solutions.

Nous ne craignons pas d'affirmer qu'il faut parfois s'opposer aux solutions préconisées par les habitants, car elles peuvent s'avérer inadaptées. Mais il faut expliciter clairement les raisons qui conduisent à ne pas retenir leurs propositions.

Par ailleurs les professionnels doivent soumettre leurs esquisses de solutions à la critique des habitants, en expliquer les fondements et s'efforcer d'intégrer les critiques pour améliorer ces propositions, jusqu'à ce qu'elles soient validées par la grande majorité des usagers. 
Élaborer et Interpréter la Demande des Habitants afin de la Problématiser

Lorsque l'on consulte les habitants, ils réagissent souvent de manière brutale, à l'emportepièce, et de façon d'autant plus virulente que leurs problèmes n’ont pas été pris en compte pendant plusieurs années. Ils tendent également à se focaliser sur des points particuliers qui les touchent personnellement, ou qui empoisonnent leur vie quotidienne: infiltrations d'eau, moisissures sur les murs, bruit des jeux d'enfants, saleté et dégradation des parties communes, etc.

Ces réactions sont normales, il importe à la fois de prendre en compte ces problèmes et de les dépasser en essayant de comprendre les usages et les modes d'habiter, les significations attachées à l'espace. Cela nécessite un travail d'élaboration de la demande en favorisant l'expression des attentes concernant le devenir des quartiers.

Par ailleurs il ne faut pas prendre à la lettre les demandes exprimées, mais interpréter leur signification, repérer les causes de leur insatisfaction. Par exemple quand les habitants se plaignent de l'insécurité et réclament un renforcement de la répression, il faut comprendre que la source de leur insécurité peut résulter de l'interaction entre des déficiences de l'aménagement de l'espace et de la gestion de l'habitat, mais aussi de leur isolement social.

Si on ne fait pas ce travail d'interprétation on peut faire de graves contre-sens sur l'origine des problèmes, sur la nature des attentes, et dans le choix des modes d'intervention.

Ce travail d'élaboration et d'interprétation revient à problématiser la demande, à déconstruire les problèmes qu'elle traduit, et à comprendre le sens dont elle est porteuse.

\section{Les Principes Sous-Tendant la Mise en Place des Dispositifs de Participation}

\section{Le Degré D'implication des Habitants: un indicateur de la communication des bailleurs ou des collectivités locales}

Au début d'un processus de concertation si seulement une dizaine de locataires sur 500 ou 1000 ménages participent aux réunions, ceci est un bon indicateur du déficit de communication entre le bailleur ou la collectivité locale et les habitants.
Notre expérience montre que l'on peut aisément associer plus de $40 \%$ des habitants à l'élaboration d'un projet, même si au départ seulement une dizaine d'habitants se sentent spontanément impliqués, dès lors que l'on s'en donne les moyens.

Mais le développement de l'implication des habitants nécessite la mise en place d'un dispositif de communication cohérent et d'une organisation rigoureuse.

Les Objectifs de L'implication des Habitants: prendre en compte leurs demandes, améliorer la communication avec le bailleur et développer les relations sociales.

L'implication des locataires dans l'élaboration de projets d'amélioration de l'habitat ou des services permet de prendre en compte leurs demandes et d'adapter en conséquence ces projets.

Mais cette implication à l'occasion des projets constitue une opportunité et un support idéal pour améliorer la communication du bailleur avec ses locataires dans la durée, dans la gestion quotidienne.

C'est également un support pour favoriser la régulation des conflits et développer les relations sociales entre les habitants.

Articuler la Concertation Institutionnelle avec les Associations et la Participation Opérationnelle Des Habitants

Il existe un débat très confus et des conflits entre les acteurs sur le fait de privilégier ou non la concertation avec les Associations de locataires, au détriment de la participation directe avec les habitants.

A notre sens ce débat n'a pas lieu d'être : les Associations de locataires ont une légitimité institutionnelle qui ne doit pas être remise en cause, et leur représentativité ne doit pas être suspectée.

Les associations doivent être consultées dans le cadre de l'élaboration globale des projets au niveau institutionnel projets sur lesquels elles doivent pouvoir donner leur point de vue, de manière à les valider ou à refuser de les approuver. En cas de refus de leur part, il appartient aux décideurs de renégocier avec elles ou de prendre seulement acte de leur opposition. 
Cette concertation institutionnelle ne doit pas empêcher la mise en place d'une participation plus opérationnelle de différents groupes d'habitants, en impliquant d'ailleurs les associations dans la conduite de ce processus.

\section{Nourrir L'élaboration du Projet de Différentes} Sources D'information sur les Attentes des Habitants

Les demandes et les problèmes formulés par les habitants diffèrent selon les modalités d'expression choisies. Au lieu d'opposer les formes $d$ 'expression individuelles et collectives, il est souhaitable de les articuler car elles portent rarement sur les mêmes objets.

Il est donc préférable de croiser les informations fournies par:

- Des enquêtes;

- Lobservation des pratiques et des usages de l'espace;

- Des entretiens avec des informateurs connaissant les demandes et les pratiques des habitants;

- Des réunions avec des petits groupes sur des problèmes particuliers qui les touchent;

- Des réunions collectives plus larges.

Organiser la Participation a une Echelle de 100 A 200 Logements, Cohérente avec L'organisation de la Vie Sociale et du Territoire

La communication et les relations sociales ne se développent pas à l'échelle de plusieurs centaines de logement. La règle d'or consiste à considérer que plus l'échelle du territoire de participation est importante, moins les habitants s'y investissent car ils ne se sentent pas impliqués par un univers de relations qui dépasse leur îlot voire leur immeuble.

Au-delà de la participation institutionnelle des associations, il faut donc organiser la participation à l'échelle de 100 ou 200 logements, rarement plus, selon un découpage cohérent avec les «unités d'habitat» existantes.

Ces unités d'habitat correspondent:

- À des unités urbaines qui sont fonction de l'organisation urbaine et de la morphologie des bâtiments;

- À des unités de vie sociale, d'usage du territoire et de relations sociales;
- À des unités d'identité sociale, unités auxquelles les habitants s'identifient (même négativement) et qui correspondent à un sentiment d'appartenance.

La participation doit en effet contribuer à renforcer ce sentiment d'appartenance et cette identification qui permettent d'étayer leur investissement dans l'habitat. Elle doit également favoriser la cohésion sociale de ces unités d'habitat, en permettant de réguler les tensions et de développer les relations de voisinage.

Articuler les Unités d'Habitat de Participation et les Territoires de Gestion

Il faut que les unités d'habitat dans lesquelles est organisée la participation correspondent à des territoires de gestion dont un gérant ou des gardiens ont la responsabilité. Ceci permet:

- Que les habitants aient des interlocuteurs communs face à leurs problèmes;

- De renforcer la communication entre les gérants ou les gardiens et les habitants;

- De renforcer les responsabilités des gardiens;

- De faire en sorte que les gardiens constituent un pôle d'identification collective contribuant à la régulation sociale et au développement de la cohésion.

Intégrer la Participation dans le Dispositif de Conduite Opérationnelle des Projets

La participation est souvent déléguée à des agents de développement social ou des prestataires qui consultent les habitants en amont de l'élaboration des projets et qui transmettent les attentes recueillies aux concepteurs. Ou bien la participation consiste à soumettre les avant-projets aux habitants, en espérant que les concepteurs en tiendront compte.

Ceci conduit souvent à ce que les attentes des habitants ne soient pas réellement prises en compte, ou ne le soient que de façon marginale. Il est en effet difficile pour les concepteurs d'intégrer des demandes qui ne s'inscrivent pas dans le processus d'élaboration des projets.

La participation doit être conduite sous la responsabilité du maître d'ouvrage, seul à même d'exiger la prise en compte des attentes des 
habitants. Concrètement cela signifie que les réunions avec les habitants doivent être présidées par le responsable désigné par le maître d'ouvrage.

Elle doit être organisée par l'équipe opérationnelle chargée de la programmation ou de la conception, toujours en présence du chef de projet désigné par le maître d'ouvrage, qui leur transmet les demandes des habitants et s'assure qu'ils les intègrent.

Ceci n'empêche pas que des prestataires ou des agents de développement sociaux assistent le maître d'ouvrage dans cette tâche, mais ils le font sous sa responsabilité et en étant intégrés à l'équipe opérationnelle.

\section{Organiser un Dispositif de Communication Rigoureux et Cohérent}

La participation des habitants est souvent gérée de manière improvisée, de manière incidente à l'élaboration des projets, alors qu'elle doit être considérée comme un élément clé de la réussite des opérations et donner lieu à l'organisation d'un dispositif de communication rigoureux et cohérent. Mais ce dispositif doit être parfaitement intégré au processus de conduite des projets comme nous l'avons évoqué précédemment.

\section{Faire Porter la Participation sur des Enjeux et des Objets Concrets}

Il est difficile de mobiliser les habitants sur la conception globale des projets. En outre si on se contente de les consulter sur la définition des grandes orientations de transformation de l'espace ils risquent d'être leurrés, car la traduction concrète d'orientations qu'ils auraient approuvé peut s'avérer en complète contradiction avec leurs attentes.

Il est à la fois plus aisé et plus efficace de faire porter la participation sur des objets très concrets, comme l'aménagement de halls, l'implantation d'aires de jeux, l'organisation de la circulation et des parkings, l'aménagement des abords d'immeubles, etc.

S'appuyer sur les Responsables et les Agents de Gestion pour Développer la Participation

De nombreux bailleurs sous-traitent à des équipes extérieures l'animation de la participation des habitants. De ce fait, ce genre de démarche, même si elle est réussie, n'a pas d'effet sur les relations entre le bailleur et le locataire. Lorsque l'équipe d'animation achève sa mission et se retire une fois le projet terminé il ne reste rien, les relations reprennent comme avant et cela génère souvent une immense déception. Les habitants se retrouvent à nouveau abandonnés et isolés.

Par contre si la participation est animée par les responsables et les agents de gestion, avec l'assistance éventuelle d'une équipe de conseil pour organiser ce processus, la participation à l'élaboration d'un projet constitue effectivement un support de développement de la communication dans la durée avec le personnel de gestion.

L'opération de participation devient alors un support de qualification des agents et de développement de leurs relations avec les habitants.

Mobiliser les Acteurs Sociaux, les Associations et les Habitants-Relais

Le développement de la participation peut s'appuyer sur les acteurs sociaux du quartier et les différentes associations, mais également sur des habitants-relais qu'il importe de repérer afin de constituer un réseau de communication.

Il faut cependant veiller à ce que la participation ne se limite à ces acteurs, qui risquent de faire écran à la participation de la majorité des habitants. Ces acteurs doivent jouer un rôle de relais de communication pour mobiliser les habitants.

Préparer et Adapter les Organisations et Qualifier les Agents a Travers la Mise en Place d'un Processus de Participation

De nombreuses opérations de participation échouent car les organisations concernées et les agents:

- Ne sont pas préparés à recevoir les critiques parfois virulentes des habitants;

- Ne savent pas écouter, entendre et prendre en compte leurs demandes;

- Ne sont pas formés à l'organisation de la concertation, à l'animation des réunions et à la négociation avec les habitants.

Il importe donc de préparer l'organisation et de former les agents afin de les mettre en capacité de recevoir les critiques, d'écouter, d'organiser le dialogue et la négociation.

Quand les organisations et les agents ne sont pas préparés à recevoir des critiques ils réagissent 
de manière défensive et tendent à se protéger en se justifiant, voire en se refermant davantage dans un fonctionnement bureaucratique.

Moyennant une assistance pédagogique, l'implication des agents dans un processus de participation constitue un excellent support d'apprentissage pour apprendre à organiser et animer des réunions, à gérer des conflits, à négocier et à développer la communication avec les habitants.

\section{Adapter le Processus de Participation en Fonction} de la Dynamique Sociale du Quartier, de son Organisation Urbaine et des Modes de Gestion

Il va de soi que le processus de participation doit être adapté en fonction de la dynamique sociale du quartier et des capacités d'expression de la population.

Les démarches à mettre en œuvre sont très différentes selon qu'il existe une forte dynamique associative, des réseaux de sociabilité importants ou bien que nous soyons dans une situation très conflictuelle ou d'isolement social d'une part importante des habitants.

Il faut également tenir compte de l'organisation urbaine du quartier et des modes de gestion, ainsi que des capacités de communication des acteurs locaux.

Les Echecs de la Participation dus a un Déficit d'organisation et de Compétence Entraînant une Dégradation de la Communication

Les responsables et les agents qui font l'expérience d'un échec, généralement dû à un manque de préparation, d'organisation et de compétence, deviennent souvent hostiles à l'égard des processus de participation.

Leurs réactions et l'insatisfaction des habitants qui en découlent entraînent souvent une dégradation de leurs relations et de leur communication.

Une Démarche de Participation Associant 300 Habitants au Réaménagement de l'espace Urbain d'un Quartier de 700 Logements (Quartier Des Hauts De Belleville - Opac de Paris)

A titre d'exemple, nous présentons un processus de participation des habitants que nous avons mis en place à la demande de l'OPAC de Paris, dans le cadre d'une opération de réaménagement des espaces urbains et des halls d'un quartier de 700 logements. Ce processus s'inscrivait dans la mission de programmation de ce réaménagement que l'OPAC de Paris nous a confiée en coopération avec l'architecte urbaniste Jean-Didier Laforgue.

Il s'agit d'un quartier d'urbanisme sur dalle, situé sur la colline de Belleville et comportant 5 niveaux de dalles, auxquelles s'ajoutent 5 niveaux de parkings souterrains. L'espace urbain de ce quartier était un véritable labyrinthe, fait de passerelles, d'escaliers en tous sens, de halls traversant, d'immenses couloirs devenus très insécurisants pour les adultes, car ils étaient parfaitement contrôlés par des groupes délinquants.

Il faut souligner le fait que cette opération a été une réussite car elle a été pilotée directement par la Direction Générale de l'OPAC qui a désigné un chef de projet très compétent assurant l'organisation de la conduite de la démarche et l'interaction entre les services internes de l'OPAC (entre la Direction, les Services de réhabilitation et les Services de Gestion).

En outre le responsable de l'Antenne de Gestion était intégré à l'équipe opérationnelle. Le programme de réaménagement a été conçu de manière à améliorer les conditions d'habitat et la dynamique sociale du quartier, mais aussi en concevant des territoires facilitant la gestion.

Le programme a été évalué systématiquement pour intégrer l'ensemble des problèmes de gestion et les gardiens ont été également consultés.

A partir d'une enquête réalisée auprès d'un échantillon d'habitants particulièrement exposés à l'insécurité, l'orientation proposée a été de réorganiser ce " magma urbain » en 6 résidences autonomes de 100 logements environ, ayant chacune son jardin propre, son entrée parking, son entrée sur rue et un gardien responsable de chaque territoire. Cette orientation permettait de supprimer la plupart des escaliers, des passerelles et des couloirs angoissants.

Par ailleurs un terrain de sport a été réaménagé avec les jeunes. 
La Participation des Habitants au Cours de la Programmation

\section{L'Échec de la Première Réunion}

Au démarrage de l'opération une réunion a rassemblé seulement une dizaine d'habitants et tout le monde était convaincu qu'il était impossible de mobiliser les habitants

\section{L'Enquête Sociologique Légère}

Une enquête a été effectuée auprès de 70 habitants vivant dans deux bâtiments particulièrement insécurisés. Elle a permis de comprendre les problèmes et les attentes des habitants de ces immeubles, et de les inviter personnellement à une réunion pour la semaine suivante.

\section{Une Réunion Test}

40 habitants sur les 70 rencontrés sont venus à cette réunion. Il s'agissait de tester leurs réactions vis à vis de la proposition de résidentialisation du quartier.

Comme leur adhésion a été quasiment unanime, nous avons poursuivi le travail de programmation sur cette base.

\section{La Participation au Cours de l'Elaboration des Projets de Résidence}

Quatre maîtres d'œuvre différents ont été retenus par l'OPAC de Paris pour élaborer les projets d'aménagement de 6 résidences et du terrain de sport.

Il convient de signaler qu'à l'issue de notre mission de programmation, nous avons assuré une mission d'assistance à la mise en œuvre auprès du chef de projet désigné par la direction de l'OPAC pour:

- Contribuer à ce que les maîtres d'œuvre respectent le programme validé par l'OPAC;

- Aider l'OPAC à organiser la participation des habitants au cours de l'élaboration des projets.

Un rôle de Médiateur - traducteur

Notre rôle consistait à assurer une médiation entre l'OPAC, les maîtres d'œuvre et les habitants de manière à:
- retraduire en terme de conception urbaine et architecturale les attentes des habitants auprès des maîtres d'œuvre

- s'efforcer d'intégrer ces attentes dans leur projet.

Une organisation des réunions de participation par résidence de 120 logements pour renforcer leur cohésion sociale et l'identité collective

Il s'agissait de concevoir les projets en fonction des attentes spécifiques de chaque groupe de résident d'une part et d'utiliser cette opportunité comme support pour renforcer leur cohésion sociale en favorisant les relations et les échanges entre eux. C'est pourquoi la participation a été organisée par résidence, échelle pertinente pour développer l'inter connaissance et les identités collectives. 30 à 50 habitants de chaque résidence ont ainsi participé aux différentes réunions.

Trois réunions d'habitants pour concevoir les projets de réaménagement de chaque résidence

Pour concevoir chaque projet de résidence, trois réunions ont été organisées par L'OPAC avec notre assistance et avec la participation active des maîtres d'œuvre.

- Une première réunion de présentation et d'explicitation du programme afin de répondre aux questions des habitants et recueillir leur réaction. Les maîtres d'œuvre se contentaient alors d'écouter les demandes afin de s'imprégner du contexte social et de prendre connaissance des attentes des habitants.

- Une seconde réunion de présentation d'un schéma d'intentions des concepteurs. A cours de cette réunion les concepteurs explicitaient leur choix et recueillaient les réactions, les critiques et les demandes de modification de l'avant-projet.

- Une troisième réunion de validation d'une esquisse d'avant-projet. Les concepteurs étaient donc amenés à modifier leur schéma de principe pour s'efforcer d'intégrer les attentes des habitants. La troisième réunion permettait de valider le projet en y apportant encore certaines retouches.

- Dans quelques cas, l'avant-projet définitif a été exposé dans les halls. 
L'implication des jeunes dans la conception d'un terrain de sport

Grâce à la médiation d'une équipe d'animation, nous avons pu associer les jeunes à la conception d'un terrain de sport, à travers des réunions directement sur le site avec le maître d'œuvre.

La mobilisation des gardiens chargés d'inviter personnellement chaque habitant aux réunions

Outre des affiches dans les halls, les gardiens étaient chargés de rencontrer chaque habitant pour leur transmettre personnellement une lettre d'invitation de l'OPAC aux réunions.

Une démarche permettant de renforcer les relations entre les habitants et leur gardien

Cette démarche s'est avérée très efficace et a permis aux gardiens de rencontrer des habitants qu'ils connaissaient peu ou pas du tout.

Un travail d'intégration des demandes des habitants par les concepteurs

A l'issue de chaque réunion avec les habitants, dans le cadre des deux premiers projets sectorisés, nous avons organisé une réunion de travail avec le maître d'œuvre concerné pour l'aider, le convaincre, ... voire l'obliger parfois à modifier son projet afin d'intégrer les attentes des habitants. Par la suite, le Chef de Projet a assuré seul la conduite du processus.

Au cours des réunions avec les habitants nous notions leurs attentes et nous les transmettions (par écrit et par oral) aux maîtres d'œuvre en les retraduisant en termes de suggestions de modification envisageable (ou indispensables) de leur projet. Certains concepteurs se sont avérés très coopératifs... d'autres étaient plus réticents, sachant que le responsable du maître d'ouvrage tranchait en dernier ressort.

Nous étions un peu les avocats de la parole des habitants dans ce travail, tout en intégrant les contraintes du maître d'ouvrage (notamment de coût et de gestion) et en nous inscrivant dans les intentions des concepteurs, dès lors qu'elles étaient fidèles à l'esprit du programme.

Nous étions dans une position inter-culturelle car nous comprenons relativement bien les enjeux, les contraintes et les modes de raisonnement des maîtres d'ouvrage, des concepteurs et des habitants.

\section{Conclusion}

En conclusion de cette note méthodologique il convient d'insister sur le fait que la participation des habitants dépend avant tout des modes de communication développés par les organisations qui assurent la gestion des quartiers, de leur capacité d'écoute et de prise en compte des attentes des habitants et de l'efficacité des dispositifs qu'ils mettent en place.

En outre les processus de participation ne doivent pas être considérés comme une concession ou une plus-value d'âme accordée conjoncturellement aux habitants. Ces processus doivent être intégrés dans le fonctionnement normal des organisations qui gèrent les quartiers et dans la conduite des projets qu'ils mènent : l'efficacité de ces processus conditionne la pertinence et l'efficacité de ces projets et des investissements qu'ils nécessitent. 